\title{
Questes
}

Revue pluridisciplinaire d'études médiévales

Journée d'étude 1 - Trier, classer, organiser | 2022

Trier, classer, organiser

\section{Entre fleur de Lys et croix de Savoie : ordonner le monde alpin sous le manteau de Marie ( $\mathrm{XV}^{\mathrm{e}}$ $\mathrm{XVI}^{\mathrm{e}}$ siècles)}

Thalie Balayn

\section{OpenEdition}

\section{Journals}

Édition électronique

URL : https://journals.openedition.org/questes/6070

DOI : 10.4000/questes.6070

ISSN : 2109-9472

\section{Éditeur}

Les Amis de Questes

\section{Édition imprimée}

Pagination : 245-257

ISSN : $2102-7188$

Référence électronique

Thalie Balayn, «Entre fleur de Lys et croix de Savoie : ordonner le monde alpin sous le manteau de Marie (xve-xvI ${ }^{e}$ siècles) », Questes [En ligne], Journée d'étude 1 - Trier, classer, organiser | 2022, mis en ligne le 31 janvier 2022, consulté le 21 février 2022. URL : http://journals.openedition.org/questes/ 6070 ; DOI : https://doi.org/10.4000/questes.6070 


\title{
Entre fleur de Lys et croix de Savoie : ordonner le monde alpin sous le manteau de Marie $\left(\mathrm{XV}^{\mathrm{e}}-\mathrm{XVI}^{\mathrm{e}}\right.$ siècles $)$
}

\author{
Thalie BALAYN
}

LUHCIE

L'image de la Vierge au manteau bénéficie depuis peu d'une attention parmi les chercheurs ${ }^{1}$. Néanmoins, peu d'enquêtes globales existent. Pour cela, il faut remonter en 1908 à la thèse de Paul Perdrizet qui propose la seule étude iconographique complète ${ }^{2}$. Malgré un corpus considérable, seules deux peintures sont évoquées pour notre terrain d'étude ${ }^{3}$. Or, depuis son enquête, nombre de Vierge au manteau ont été découvertes dans le Dauphiné et l'ancien Duché de Savoie, dont beaucoup ont échappé à une analyse scientifique, mais aussi à la simple reconnaissance de ce patrimoine. Cette communication se propose d'offrir

\footnotetext{
${ }^{1}$ Quelques publications récentes sont parues, notamment l'étude monographique d'Hélène Millet et Claudia Rabel, La Vierge au manteau du Puy-en-Velay : un chefd'œuvre du gothique international (vers 1400-1410), Lyon, Fage, 2011. Pour une approche régionale de l'Italie du Nord aux XIV et $\mathrm{XV}^{\mathrm{e}}$ siècles, voir Tommaso Castaldi, La Madonna della Misericordia. L'iconografia della Madonna della Misericordia e della Madonna delle frecce nell'arte di Bologna e della Romagna nel Tre e Quatrocento, Imola, La Mandragora, 2011. Pour une étude thématique du sujet, voir Jérôme Baschet, Iconographie médiévale, Paris, Gallimard, 2008, p. 274-279. Pour une étude historique du thème, voir le travail de Christa Belting-Ihm, « Sub Matris Tutela », dans Untersuchungen zur Vorgeschichte der Schutzmantelmadonna, Heidelberg, Carl Winter, 1976, p. 8-110.

${ }^{2}$ Paul Perdrizet, La Vierge de miséricorde, étude d'un thème iconographique, Paris, Albert Fontemoing, 1908. L'auteur recense 258 représentations de la Vierge au manteau pour l'ensemble de l'Occident médiéval - dont 64 en France et 119 en Italie réalisées avec des techniques et supports divers.

${ }^{3}$ Ibid., p. 87 et 175 . Il s'agit des œuvres de Nice et de Saint-Étienne-de-Tinée.
} 
un éclairage neuf sur cette iconographie dans l'ancien duché de $\mathrm{Savoie}^{4}$ et en Dauphiné, à la fin de l'époque médiévale 5 .

L'étude de la Vierge au manteau est particulièrement singulière sur le plan formel et sémantique, que nous aborderons dans un premier temps. Son étude nous mène indiscutablement sur le terrain de l'anthropologie médiévale en mobilisant les questions des croyances populaires, du contexte historique et spatial, mais aussi des commanditaires, que nous traiterons dans un second temps. De la sorte, cette communication tend à illustrer l'ordonnancement du monde médiéval au travers de l'organisation des fidèles sous le manteau, centrée sur un espace singulier, le monde alpin, dans lequel la Vierge de miséricorde revêt un caractère spécifique ${ }^{6}$.

\footnotetext{
${ }^{4}$ À cette époque, le duché de Savoie s'étend de la Bresse au Pays de Vaud, puis de Nice au Piémont, jusqu'au Val d'Aoste, ce qui recouvre les actuels départements de la Savoie, Haute-Savoie, Hautes-Alpes, Alpes-Maritimes pour le versant français ; les provinces du Val d'Aoste, du Val de Suse, de la plaine du Pô et de l'Imperia pour le versant italien.

${ }^{5}$ Paul Deschamps, «La Vierge au manteau dans les peintures murales en Savoie et Dauphiné », dans Comité des travaux historiques et scientifiques. Actes du $85^{e}$ Congrès National des Sociétés savantes. Section d'archéologie. Séance du samedi 9 avril. Chambéry-Annecy 1960, Paris, Imprimerie Nationale, 1962, p. 145-150. Son étude expose avant tout la théorie des orants sous le manteau, qui seraient les chevaliers morts pour la bataille de Verneuil. Pour une approche comparative stylistique des peintures savoyardes, voir Nicolas Schätti, « La Vierge de miséricorde. Contribution à l'étude de la peinture dans l'ancien duché de Savoie », Nos monuments d'art et d'histoire, 39, 1988, p. 72-80. Enfin, pour une étude de filiation entre une peinture savoyarde et une ponote, voir Hélène Millet, "Quels maillons entre "la Vierge au manteau" du Puy-enVelay et celle du château de Fénis ? », dans Entre France \& Italie. Mélanges offerts à Pierrette Paravy. Vivacité et rayonnement d'une rencontre, dir. Laurence Ciavaldini Rivière, Anne Lemonde-Santamaria et Ilaria Taddei, Grenoble, Presses Universitaires de Grenoble, 2009, p. 47-57.

${ }^{6}$ Tous les auteurs s'accordent sur la hiérarchie du thème de la Vierge mantelée. La seule étude comparative de cette iconographie entre Dauphiné et maison de Savoie demeure l'article de Paul Deschamps, «La Vierge au manteau dans les peintures murales en Savoie et Dauphiné », art. cit. Toutefois, son analyse est centrée sur la peinture de Laval afin d'émettre une hypothèse sur l'identité des priants sous le manteau, ainsi que sur les possibles commanditaires de l'œuvre. Les peintures savoyardes de Fénis et d'Hauteluce sont également mentionnées, mais sans une analyse approfondie des priants sous le manteau.
} 
Vingt représentations peintes de la Vierge au manteau occupent, à notre connaissance, les territoires médiévaux du Dauphiné et de l'ancien duché de Savoie ${ }^{7}$. De ce corpus iconographique, nous n'en étudions volontairement qu'un échantillon afin de nous pencher sur les éléments les plus parlants de cet axe de recherche. Pour le Dauphiné, quatre représentations sont retenues : celles du Genevray-de-Vif, de Laval, et les deux peintures perdues de Grenoble et de Saint-Antoine-1'Abbaye ${ }^{8}$. Pour les cuvres savoyardes, nous retiendrons cinq représentations: les miniatures des deux livres d'Heures, la peinture de Fénis et les deux de Genève (dont une a disparu ${ }^{9}$ ). Avant d'étudier le corpus d'images proposé, il convient de commencer par la définition de cette image et d'en examiner plusieurs aspects.

\section{Organiser, entre le monde divin et le monde terrestre}

Le thème de la Vierge au manteau est né à la fin du Moyen Âge et a connu un vif succès ${ }^{10}$. Le contexte troublé de la guerre de Cent Ans, ainsi que la pandémie de peste qui frappa l'Occident, favorisèrent une diffusion

\footnotetext{
${ }^{7}$ Sept représentations de la Vierge au manteau se retrouvent en Dauphiné : Laval, Genevray-de-Vif, Claveyson, Casteldelfino et Salbertrand. Celles de Saint-Antoinel'Abbaye et de Grenoble ont disparu. Dans le duché de Savoie sont conservées 13 Vierge au manteau et une qui a disparu à Genève. Trois autres sont dans un état fragmentaire : celles d'Avigliana, de Bousson et de la Sacra di San Michele. Deux autres peintures se trouvent dans des livres d'Heures ayant appartenu au duc de Savoie (voir la note 9 pour les références). Les peintures sont visibles à Fénis, Genève, Hauteluce, Saint-Étienne-de-Tinée, Saluces, Villafalletto, et deux à Nice.

${ }^{8}$ Une description dans le Registre Delphinal de Mathieu Thomassin permet de les restituer. Voir Mathieu Thomassin, Registre Delphinal, $\mathrm{XV}^{\mathrm{e}}-\mathrm{XVI}^{\mathrm{e}}$ siècles, Grenoble, BM, U.909, fol. 91r.

${ }^{9}$ Les Heures du comte de Piémont sont conservées à la Stadtbibliothek de Stuttgart, HB.I.175, tandis que les Heures de Louis de Savoie sont à la BnF, lat. 9473. La peinture disparue de Genève est accessible grâce à la description de François de Bonivard. Voir François de Bonivard, Chroniques de Genève, t. 2, Genève, Dunant, 1831, p. 158.

${ }^{10}$ Voir Paul Perdrizet, La Vierge de miséricorde, op. cit., p. 107-193 et Émile Mâle, $L$ 'Art religieux de la fin du Moyen Âge en France. Étude sur l'iconographie de la fin du Moyen Age et sur ses sources d'inspiration, Paris, Armand Colin, 1995, p. 198-205.
} 
très rapide de l'image ${ }^{11}$. Toutefois, sa fortune fut de courte durée, étant donné le succès de l'humanisme et les avancées de la Renaissance italienne $^{12}$.

L'image s'articule autour de la figure de la Vierge, entourée d'orants. Elle est debout, de plain-pied, au centre de la composition et fait face au fidèle en écartant de ses bras les pans de son manteau ${ }^{13}$. Elle est d'une taille colossale, tandis qu'une multitude de petits personnages sont tournés vers elle, les mains jointes ${ }^{14}$. Cette image renvoie à la conception du monde propre à l'homme médiéval. La taille immense de la Vierge tout comme sa position frontale marquent sa sainteté et son pouvoir d'intercesseur auprès de Dieu, tandis que la petite taille et la figuration de profil des suppliants rappellent leur condition humaine de simples mortels ${ }^{15}$. Cet ordonnancement du monde médiéval est inscrit dans l'image par sa composition de forme pyramidale : le chef de Marie en constitue le sommet tandis que les fidèles forment sa base.

Le manteau forme une séparation qui scinde horizontalement la composition en deux registres distincts. De manière métaphorique, cette ligne divise le monde céleste du domaine terrestre. Toutes les figures dépassant la limite supérieure de ce manteau sont auréolées en signe de sainteté $^{16}$. Au contraire, les orants, sans auréole, sont placés sous le manteau de la Vierge et confinés au monde terrestre ${ }^{17}$. Cette dichotomie

\footnotetext{
${ }^{11}$ Pour les origines du thème, voir la thèse de Nancy Hubbard, Sub Pallio: The sources and Development of the Iconography of the Virgin of Mercy, thèse de doctorat, Northwestern University, 1984.

${ }^{12}$ Louis Réau, Iconographie de l'art chrétien. Tome 2 : Iconographie de la Bible. Livre 2 : Nouveau Testament, Paris, Presses Universitaires de France, 1957, p. 118.

${ }^{13}$ Paul Perdrizet, La Vierge de miséricorde, op. cit., p. 198-201.

${ }^{14}$ François Garnier, Le langage de l'image au Moyen Âge. Signification et symbolique, Paris, Le Léopard d'Or, 1982, voir notamment chap. 5.

15 Ibid.

${ }^{16}$ Hélène Millet et Claudia Rabel, La Vierge au manteau du Puy-en-Velay..., op. cit., p. 138.

${ }^{17}$ Ibid.
} 
entre les deux mondes se retrouve de façon semblable dans les représentations du Jugement dernier, comme le montrent les fresques du $\mathrm{Xv}^{\mathrm{e}}$ siècle de la chapelle Notre-Dame-des-Fontaines de la Brigue ${ }^{18}$.

De la sorte, la figure de la Vierge fait plastiquement le lien entre ces deux mondes, car Marie est reconnue par l'Église comme jouant le rôle de «pont entre le ciel et la terre ${ }^{19}$ ». Cette configuration renvoie aux notions d'intercesseur et d'échelle céleste, Marie étant le symbole qui assure à la fois la séparation et la liaison entre les deux mondes ${ }^{20}$. Un parallèle se crée entre l'image de l'Église et celle de la Vierge. En effet, l'Église est considérée comme la mère des fidèles, et la Vierge est mère du Christ. Ainsi, dès le XII ${ }^{\mathrm{e}}$ siècle, Marie prend l'appellation de Mater Ecclesia et devient l'allégorie personnifiée de l'Église chrétienne ${ }^{21}$. Le rouleau d'Exultet de l'abbaye du Mont-Cassin représente la Mater Ecclesia ${ }^{22}$. Une figure féminine monumentale se tient debout en étendant ses bras. À sa dextre se tient une foule identifiée par une inscription Clerus, tandis qu'à sénestre se trouve le Populus. Cette composition rappelle, sans conteste, celle de la Vierge au manteau ${ }^{23}$.

${ }^{18}$ On peut noter des similarités dans la structure des deux thèmes iconographiques. Les deux images sont composées de registres superposés distinguant les mondes céleste et terrestre. Dans le Jugement dernier, le Christ siège au milieu dans la partie supérieure avec la cour céleste, tandis qu'au registre inférieur l'humanité attend son jugement. D'autre part, une dichotomie est lisible avec à dextre les élus et à sénestre les réprouvés, séparés par saint Michel pesant les âmes.

19 Jean Delumeau, Rassurer et protéger. Le sentiment de sécurité dans l'Occident d'autrefois, Paris, Fayard, 1989, p. 281.

${ }^{20}$ Christian Heck, L'Échelle céleste dans l'art du Moyen Âge, Paris, Flammarion, 1997, p. 189. Voir également Jean Delumeau, Rassurer et protéger, op. cit., p. 281.

${ }^{21}$ Gertrud Schiller, Ikonographie des Christlichen Kunst, t. IV, livre 2: Maria, Gütersloch, Mohn, 1980, p. 194.

22 Ibid. Voir aussi Hélène Millet et Claudia Rabel, La Vierge au manteau du Puy-enVelay..., op. cit., p. 137.

23 Ibid. La Vierge de miséricorde se trouve être un syncrétisme des thèmes iconographiques du répertoire de Marie. Notamment, le couronnement de la Vierge, qui est un sujet très ancien, est relaté par Grégoire de Tours au VI siècle. Sur ce point, voir Gaston Duchet-Suchaux et Michel Pastoureau, La Bible et les saints. Guide iconographique, Paris, Flammarion, 1990, p. 93. Sur le thème de la Vierge à l'Enfant, 
La figure de Marie marque aussi une dichotomie verticale, séparant distinctement le groupe de droite et celui de gauche. En effet, la foule a priori unie des priants se trouve en réalité scindée en deux. Aussi, l'assemblée des personnages sous le manteau semble, à première vue, désordonnée. Pourtant, leur disposition sous le manteau n'a pas été retenue au hasard ${ }^{24}$. En effet, une première classification peut être faite entre les clercs et les laïcs, eux-mêmes identifiés par un «personnage-type » qui définit son état, allant du pape au simple moine et de l'empereur jusqu'au paysan $^{25}$. La seconde classification s'opère au niveau du groupe sous le manteau. Lorsque Marie protège la chrétienté tout entière, on parle du type de la Mater Omnium, ou «mère de tous », identifié par Paul Perdrizet ${ }^{26}$. En 1957, Louis Réau réétudie les types de Vierge au manteau élaborés par ses prédécesseurs ${ }^{27}$. Il en retient trois : le premier est la Mater Omnium, qui est le plus répandu ; le deuxième est la «protectrice d'une collectivité », qui abrite sous son manteau un groupe plus restreint mais homogène, comme une famille, un ordre religieux ou une confrérie; et le troisième est le type Mater Unis, protégeant un unique fidèle ${ }^{28}$. Ces typologies

qui est très largement répandu depuis l'époque romane, voir notamment Hélène Millet et Claudia Rabel, La Vierge au manteau du Puy-en-Velay..., op. cit., p. 59. Sur le thème de la Mater Ecclesia (ou Vierge-Église), qui est connu depuis le $\mathrm{XI}^{\mathrm{e}}$ siècle, voir Marie. Le culte de la Vierge dans la société médiévale, dir. Dominique Iogna-Prat, Eric Palazzo et Daniel Russo, Paris, Beauchesne, 1996. De manière générale, sur l'iconographie mariale, voir Louis Réau, Iconographie de l'art chrétien, op. cit., ou son pendant germanique : Gertrud Schiller, Ikonographie des Christlichen Kunst, op. cit., t. 4, livre 2 .

${ }^{24}$ Hélène Millet et Claudia Rabel, La Vierge au manteau du Puy en Velay..., op. cit., p. 21.

${ }^{25}$ Louis Réau, Iconographie de l'art chrétien, op. cit., p. 117.

${ }^{26}$ Paul Perdrizet, La Vierge de miséricorde..., op. cit., p. 150-193.

${ }^{27}$ En effet, Maurice Vloberg détermine un type : celui de la Vierge abritant un seul priant, qui est plus tardif (vers le $\mathrm{XVI}^{\mathrm{e}}$ siècle) : voir Maurice Vloberg, La Vierge, notre médiatrice, Grenoble, Arthaud, 1938, p. 110-128. Voir aussi Louis Réau, Iconographie de l'art chrétien, op. cit., p. 110-128.

${ }^{28}$ Ibid., p. 117-118. 
permettent de caractériser les images du corpus et de proposer les quelques hypothèses qui suivent.

\section{Hiérarchie et légitimité des orants alpins}

La société du Moyen Âge est fondée sur une séparation des états que nous retrouvons ici tous protégés par la Vierge ${ }^{29}$. Toutefois, chaque orant tient une place définie sous cette protection.

Premièrement, la répartition des groupes sous le manteau n'est pas fortuite. En effet, le groupe des clercs occupe la place à la droite de la Vierge. Or le côté droit est une place privilégiée, celle des élus, impliquant la prééminence systématique des religieux sur les laïcs ${ }^{30}$. Deuxièmement, nous remarquons que les plus hauts dignitaires se retrouvent au plus près de la Vierge et surtout au premier plan de la composition. Pour le groupe des laïcs, les premiers rangs sont occupés par l'empereur et le roi, suivis de la noblesse, de la bourgeoisie et enfin du tiers état. Quant au groupe antagoniste, celui des clercs, la composition redouble cette même hiérarchie : au plus près de la Vierge, nous retrouvons le souverain pontife de l'Église, le pape, suivi des cardinaux et des évêques, jusqu'aux plus simples des clercs $^{31}$.

\footnotetext{
${ }^{29}$ Pour une approche générale, voir Georges Duby, Les Trois Ordres ou l'imaginaire du féodalisme, Paris, Gallimard, 1978. Voir aussi, pour une vision plus locale, Anne Lemonde, Le temps des libertés en Dauphiné. L'intégration d'une principauté à la couronne de France (1349-1408), Grenoble, Presses Universitaires de Grenoble, 2002. ${ }^{30}$ Hélène Millet et Claudia Rabel, La Vierge au manteau du Puy-en-Velay..., op. cit., p. 124.

${ }^{31}$ Dans nos exemples, seuls les séculiers sont représentés. Toutefois, le thème peut servir pour les réguliers, comme le panneau de Duccio, à la Pinacothèque Nationale de Sienne (Italie), qui représente Marie protégeant sous son manteau des Franciscains (1285). Les attributs permettent de situer chaque membre dans son état. Par exemple, le pape est coiffé du trirègne ; l'évêque porte la mitre ; les cardinaux sont vêtus de rouge et portent des chapeaux cardinalices; les femmes distinguées sont coiffées d'une cornette avec voile, tandis que les labours portent de simples tuniques.
} 
Hélène Millet et Claudia Rabel soulèvent une question intéressante concernant cette « société très choisie ${ }^{32}$ ». En effet, la Vierge « abrite une proportion de nobles qui n'a rien de commun avec la réalité ${ }^{33} »$. Si nous regardons les exemples de Fénis ou encore de Genève, nous nous rendons compte que la proportion des hauts dignitaires vis-à-vis des laboratores est démesurément importante, contrairement à la société médiévale, qui comportait en réalité bien plus de paysans que de notables. Ce thème de la Mater omnium vise à dépeindre une conception du monde qui est propre à l'homme médiéval.

Force est de constater que le thème de la Vierge mantelée insiste sur la représentation des visages. Généralement, les faciès des orants sont des archétypes représentant chaque état de la société, probablement afin que le fidèle s'identifie et discerne rapidement les figures peintes.

Les peintures savoyardes de Genève et de Fénis, ainsi que les deux livres d'Heures, présentent une tendance commune. Ainsi, le premier plan offre des portraits présumés des commanditaires et non des archétypes. Derrière eux, des nobles, des bourgeois puis les laboratores semblent être représentés sans individualité. Dans les Heures du comte de Piémont, Clément Gardet croit reconnaître des portraits des membres de la maison de Savoie ${ }^{34}$. Il identifie le duc Amédée VIII, sous les traits d'un prince vêtu d'un manteau rouge doublé d'hermine ${ }^{35}$. Ce manteau répondrait aux caractéristiques du rang ducal énoncé par Amédée lui-même dans les

\footnotetext{
32 Cf. Hélène Millet et Claudia Rabel, La Vierge au manteau du Puy-en-Velay..., op. cit., p. 130.

${ }^{33} \mathrm{Ibid}$.

${ }^{34}$ Clément Gardet, De la peinture du Moyen Âge en Savoie. Tome IV: Un livre d'Heures du comte de Piémont futur duc Amédée IX de Savoie, Annecy, Gardet, 1981, p. 19.

${ }^{35}$ Ibid.
} 
Statua Sabaudiae ${ }^{36}$. Quant à la femme vêtue d'une robe verte, l'auteur reconnaît la princesse Marguerite de Savoie, qui est la dernière fille d'Amédée VIII ${ }^{37}$. L'auteur discerne aussi des figures de la famille ducale sous le manteau de Marie dans un autre manuscrit : les Heures de Louis de Savoie, datées des années 1455-1460, montrent au premier plan un orant brun vêtu d'une tunique courte et de chausses ; il pourrait s'agir de Louis de Savoie (fils d'Amédée VIII), avec à ses côtés son épouse Anne de Chypre, vêtue d'une robe rebrodée de feuilles d'or ${ }^{38}$. Par conséquent, le duc de Savoie et les siens occupent les places situées au plus près de la Vierge et se rendent identifiables par des attributs et des portraits présumés.

La peinture murale de la Vierge au manteau du temple Saint-Gervais de Genève, datée des années 1447-1449, est située dans une chapelle latérale de l'édifice (fig. 1). Au pied de la Vierge, un pape est reconnaissable grâce au manteau et au trirègne ${ }^{39}$. Il aurait pu s'agir de n'importe quelle représentation archétypale du pape. Cependant, la répétition du motif du blason de la maison de Savoie le long de sa chape dévoile, en croisant la datation de l'œuvre, une autre réalité. Il s'agit de Félix V, qui prend place au plus près de la Vierge. Félix V, qui fut élu pape en 1439 au concile de Bâle et qui fut contesté par une partie des pères du Concile, cherchait peut-être à se procurer la protection de la Vierge ${ }^{40}$. Une

\footnotetext{
${ }^{36}$ Ibid. Voir aussi Marie-José de Belgique, La Maison de Savoie : Amédée VIII le duc qui devint pape, t. 3, Paris, Albin Michel, 1962, p. 59. Les Statua Sabaudiae sont des lois territoriales qui régissent les droits et les devoirs des sujets dans toute la Savoie.

${ }^{37}$ Clément Gardet, De la peinture du Moyen Âge en Savoie, t. IV, op. cit., p. 20-22.

${ }^{38} \mathrm{Ibid}$.

${ }^{39}$ Id., De la peinture au Moyen Âge en Savoie, t. I : du XI au XVe siècle, Annecy, Gardet, 1965, p. 50-51. L'édifice prend l'appellation de temple, car cette ville est devenue protestante au $\mathrm{XVI}^{\mathrm{e}}$ siècle.

40 Ibid. Félix V n'était pas reconnu comme un pape légitime par certains cardinaux. Voir Histoire du Christianisme : des origines à nos jours. Tome VI : Un temps d'épreuves (1274-1449), dir. André Vauchez, Jean-Marie Mayeur et Charles Pietri, Paris, Desclée-Fayard, 1990 ; Hélène Millet et Claudia Rabel, La Vierge au manteau du Puy-en-Velay..., op. cit., p. 126.
} 
autre peinture de Marie mantelée à Genève est connue grâce aux descriptions de François de Bonivard. Nous apprenons que parmi les orants sous le manteau, le procureur de l'hôpital de Genève, Jean Nergaz, «y estoient pourtraict au vif ${ }^{41} \gg$.

À Fénis, une Vierge protectrice orne la chapelle privée de la demeure castrale. Nous retrouvons auprès de la Vierge miséricordieuse la représentation du maréchal de Savoie Boniface de Challant ${ }^{42}(\dagger 1426)$, commanditaire des réaménagements du château de cette ville et de ses peintures murales. Il est représenté les cheveux blancs et avec un visage accusant un âge avancé. Néanmoins, ses traits sont harmonieux et réguliers, avec un front lisse et des mains délicates, qui peuvent laisser croire à un possible portrait idéalisé ${ }^{43}$.

Le corpus des peintures savoyardes est composé d'œuvres relevant autant du monde religieux que du monde laïc. En effet, plusieurs représentations de la Vierge mantelée figurent dans des églises, des chapelles, mais aussi dans des demeures privées (au château de Fénis et dans les manuscrits de la famille ducale), sur des supports variés comme la peinture sur mur, sur panneaux ou encore sur parchemin. Cette appropriation du thème religieux par les laïcs peut expliquer cette intrusion des portraits au sein de l'image pieuse. De plus, les images savoyardes montrent très régulièrement l'empereur, qui est le suzerain des comtes et des ducs de Savoie ${ }^{44}$.

\footnotetext{
${ }^{41}$ François de Bonivard, Chroniques de Genève, op. cit., p. 159.

${ }^{42}$ Hélène Millet, "Quels maillons entre "la Vierge au manteau" du Puy-en-Velay et celle du château de Fénis ? », art. cit., p. 49-51. La peinture murale date de 1415 environ.

${ }^{43}$ Ibid., p. 50. Nous n'évoquons pas l'homme quasi de dos vêtu de blanc, car son identification porte encore à discussion.

${ }^{44}$ Marie-José de Belgique, La Maison de Savoie : Amédée VIII le duc qui devint pape, op. cit.
} 
La représentation de la Vierge mantelée est tout autre dans une principauté voisine. À première vue, le Dauphiné présente des exemples de Vierge mantelée similaires sur le plan iconographique. Toutefois, à l'inverse de leur voisin savoyard, les œuvres dauphinoises sont exécutées uniquement en peinture murale et pour des lieux de culte ${ }^{45}$.

Pour cette province, le motif des fleurs de lys, symbole de la monarchie française, est identifiable, comme en témoignent les arrièreplans des peintures du Genevrey-de-Vif et de Laval ${ }^{46}$. D'un point de vue historique, le Dauphiné est rattaché à la couronne française depuis le transport en 1349, et les vierges mantelées en question sont postérieures à cet événement ${ }^{47}$. Lorsque la fleur de Lys est absente, un autre attribut royal est figuré : le manteau doublé d'hermine, dont la Vierge peinte à Laval est pourvue $^{48}$ (fig. 2). Les peintures disparues décrites dans le texte de Thomassin, sont une pièce maîtresse quant à la définition de la typologie des œuvres dauphinoises. En effet, les orants rassemblés sous le manteau sont uniquement «les chevaliers du Dauphiné tombés à la bataille de Verneuil $^{49} »$. Leur présence est la preuve d'une communauté restreinte

\footnotetext{
${ }^{45}$ Cette hypothèse doit être envisagée avec prudence, car nous nous appuyons sur les vestiges parvenus jusqu'à nous.

${ }^{46}$ Michel Pastoureau, Une histoire symbolique du Moyen Âge occidental, Paris, Seuil, 2004, p. 114-120. D'après Paul Deschamps, « La Vierge au manteau dans les peintures murales en Savoie et Dauphiné », art. cit., p. 149, la fleur de lys rappellerait les armes de la famille Allemand «de gueules, semé de fleurs de lys d'or, à la bande d'argent brochant sur le tout ». C'est sans compter que cette famille est puissante (possessions territoriales nombreuses en Dauphiné) et surtout une des alliées les plus proches du Dauphin depuis le XIII ${ }^{\mathrm{e}}$ siècle, ce qui peut expliquer les armoiries de cette illustre famille.

${ }^{47}$ Histoire du Dauphiné, dir. Bernard Blig, Toulouse, Privat, 1973, p. 161. Pour une approche récente sur la question du transport du Dauphiné, voir Anne Lemonde, Le Temps des libertés en Dauphiné, op. cit., notamment ch. 1, p. 14-46.

${ }^{48}$ Mentionnons également les œuvres de Claveyson et de Salbertrand, où l'on trouve un manteau doublé d'hermine. Sur les sept exemples dauphinois, quatre intègrent des symboles monarchiques. Casteldelfino n'en a pas, et les deux du diocèse de Grenoble, ayant disparu, ne permettent pas de le vérifier.

${ }^{49}$ Mathieu Thomassin, Registre Delphinal, op. cit., fol. 91r. La bataille de Verneuil eut lieu en 1424, en Normandie. Elle est l'une des nombreuses guerres de la Guerre de
} 
abritée sous le manteau de Marie. Les cas peints du Genevrey-de-Vif et de Laval semblent représenter les habitants des villages respectifs, car les figures stéréotypées et la hiérarchie des états semblent absentes ou, du moins, plus diluées ${ }^{50}$ (fig. 3). Selon la définition de Louis Réau, Marie au manteau dans cette principauté relève du type «protectrice d'une collectivité ».

Les cas du Dauphiné et de la maison de Savoie montrent ici les variations sur un même thème iconographique. Le Dauphiné expose toute la « fierté d'une principauté ${ }^{51} »$ vis-à-vis du Dauphin et de la couronne de France. Cette appartenance est appuyée au travers des motifs de la fleur de lys et/ou du manteau royal. Cette typologie semble mettre en évidence des différences, voire des rivalités anciennes entre les deux voisins. Elles sont caractérisées par le type «protectrice d'une collectivité », ainsi que par l'absence de l'empereur et du pape dans les peintures dauphinoises, tandis que les œuvres conservées en terre savoyarde présentent le type de la Mater Omnium et intègrent la figure de l'empereur.

Cent Ans et se solda par une défaite française, dans la lignée des batailles de Crécy (1346), Poitiers (1356) ou encore Azincourt (1415). Pour une approche générale, voir l'ouvrage récent de Nicolas Lemas, La Guerre de Cent Ans, Malakoff, Armand Colin, 2017.

${ }^{50}$ En outre, l'exemple du Genevrey-de-Vif permet l'observation des armoiries d'une famille. Nous remercions le professeur René Verdier de nous avoir fait part de son hypothèse. Ses recherches dans l'Inventaire Marcellier l'ont conduit sur l'évocation d'une famille nommée Cor, propriétaire de terres dans le «Graisivaudan » et au «Genevrey-de-Vif». Nous pouvons supposer qu'il s'agit d'un portrait du commanditaire agenouillé auprès de cet écu orné d'un cor. Voir Inventaire Marcellier, $\mathrm{XV}^{\mathrm{e}}$ siècle, Grenoble, BM, U.928, fol. 71v et Paul Deschamps, «La Vierge au manteau dans les peintures murales en Savoie et Dauphiné », art. cit., p. 130. L'auteur pense que les priants peints à Laval sont les quatre chevaliers de la famille Allemand morts à la bataille de Verneuil. Toutefois, le manque d'attribut renvoyant à l'iconographie des chevaliers ne nous permet pas de l'affirmer avec certitude, et nous penchons davantage pour une représentation des villageois et du commanditaire de l'œuvre.

${ }^{51}$ Cf. Anne Lemonde, Le Temps des libertés en Dauphiné, op. cit., p. 341. 
L'insertion des portraits sous le manteau de la Vierge témoigne de l'intérêt croissant pour l'individu à cette période, auquel le duché de Savoie semble sensible. Cette réceptivité peut s'expliquer grâce à la proximité avec l'Italie et par là avec l'idéal renaissant, qui va de pair avec l'art des portraits. Dans le Dauphiné, la distinction ne s'opère pas de la même manière, puisque les fidèles sont peu identifiables et passent par des portraits de groupe, comme l'indique le témoignage de Mathieu Thomassin à propos des chevaliers dauphinois morts à Verneuil. Surtout, sa description n'évoque pas de "pourtraict au vif », contrairement aux Chroniques de François de Bonivard ${ }^{52}$. La composition de cette image, qui est similaire d'une principauté à l'autre, rend état de l'organisation du monde médiéval, grâce, d'une part, à une lecture verticale et pyramidale, couplée, d'autre part, d'une lecture horizontale rendue par la dichotomie, hiérarchisant ainsi les fidèles entre eux et dans l'espace. C'est d'un point de vue sémantique que les variantes s'opèrent, avec le type d'orants qui sont rassemblés en son sein.

Notre conclusion ouvre sur une autre question, celle du rôle du commanditaire. En effet, la même image pieuse se trouve instrumentalisée au profit de son lieu de production et de son mécène. Cela est le témoignage indéniable que la commande d'œuvres est un prétexte pour affirmer son lignage, mais aussi pour revendiquer des liens de suzeraineté. Dès lors, cette recherche serait intéressante à mener sur d'autres principautés ou états médiévaux.

\footnotetext{
${ }^{52}$ François de Bonivard, Chroniques de Genève, op. cit., p. 158.
} 


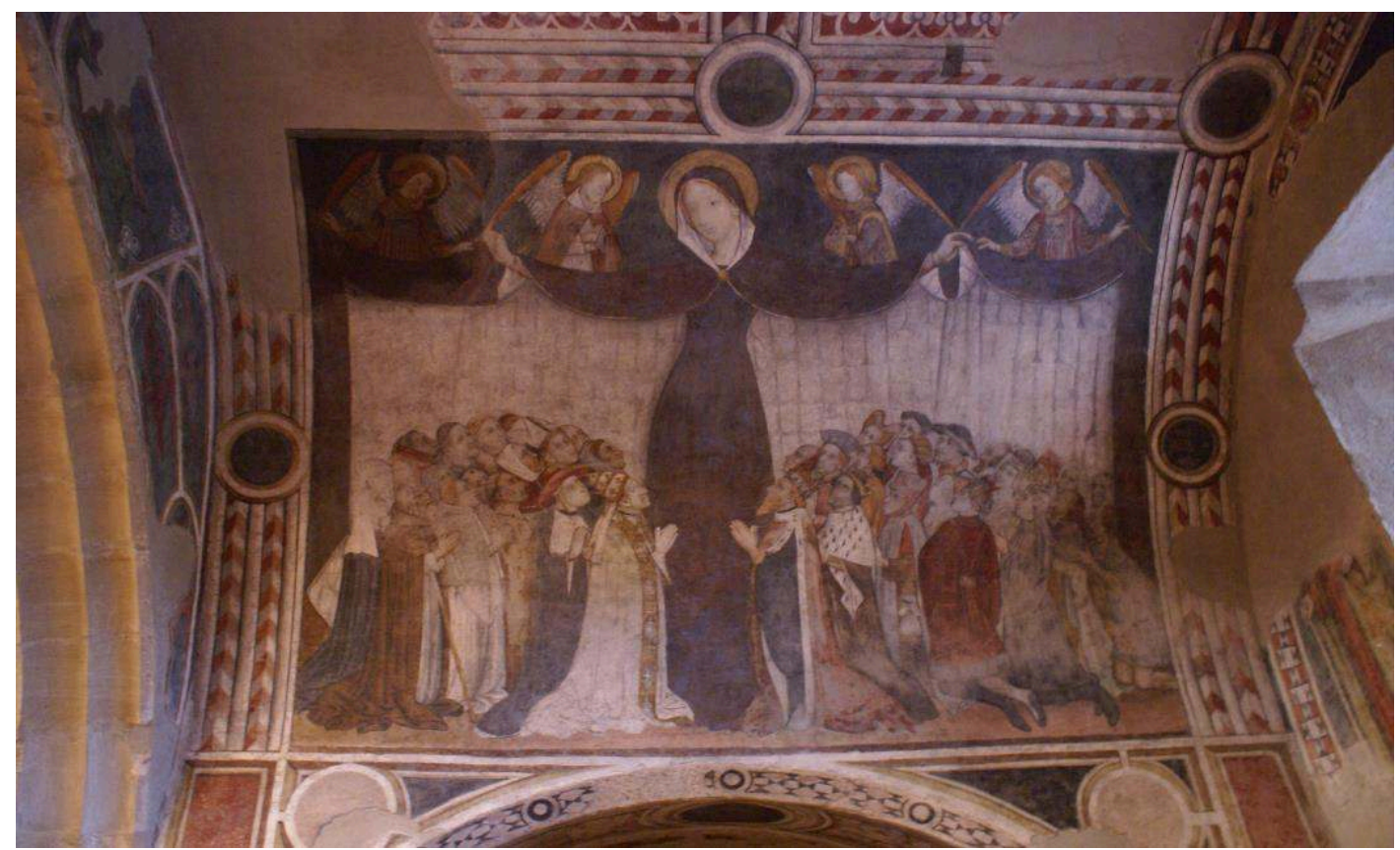

Fig. 1. Vierge au manteau. Peinture murale, a fresco, 300 x 400 cm, entre 1447-1449, Attribue a Giacomo Jaquerio, Temple Saint-Gervais, GeneVe, Suisse. Photographie de L'AUTEUR.

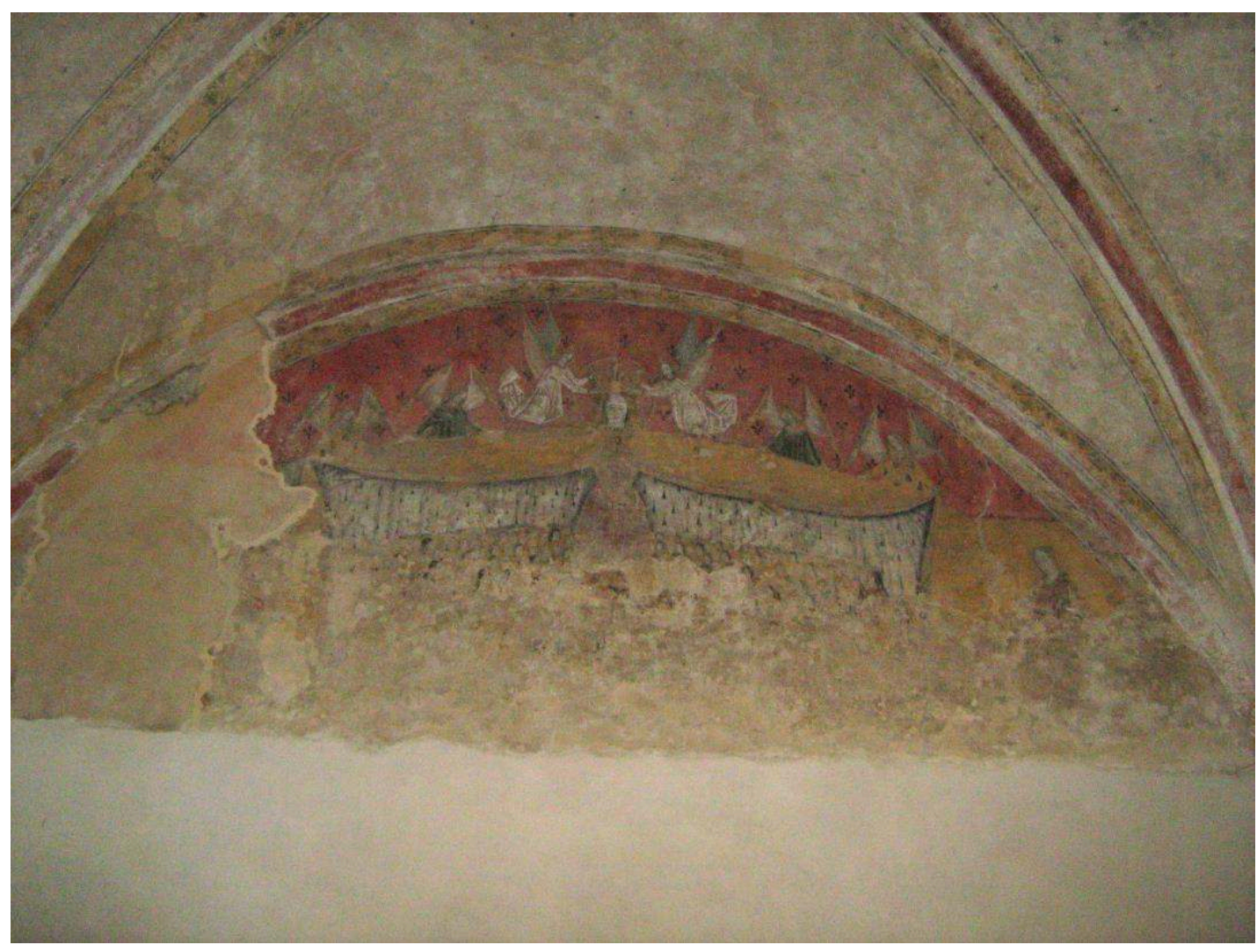

Fig. 2. Vierge au manteau. Peinture murale, barbotine de ChauX et de sable aVeC de l'huile De lin, 410 x 750 CM, IIE moitie du XVe SieCle, Anonyme, Chapelle des Alleman, Laval, France. Cliche Andre Ducluzeaux 


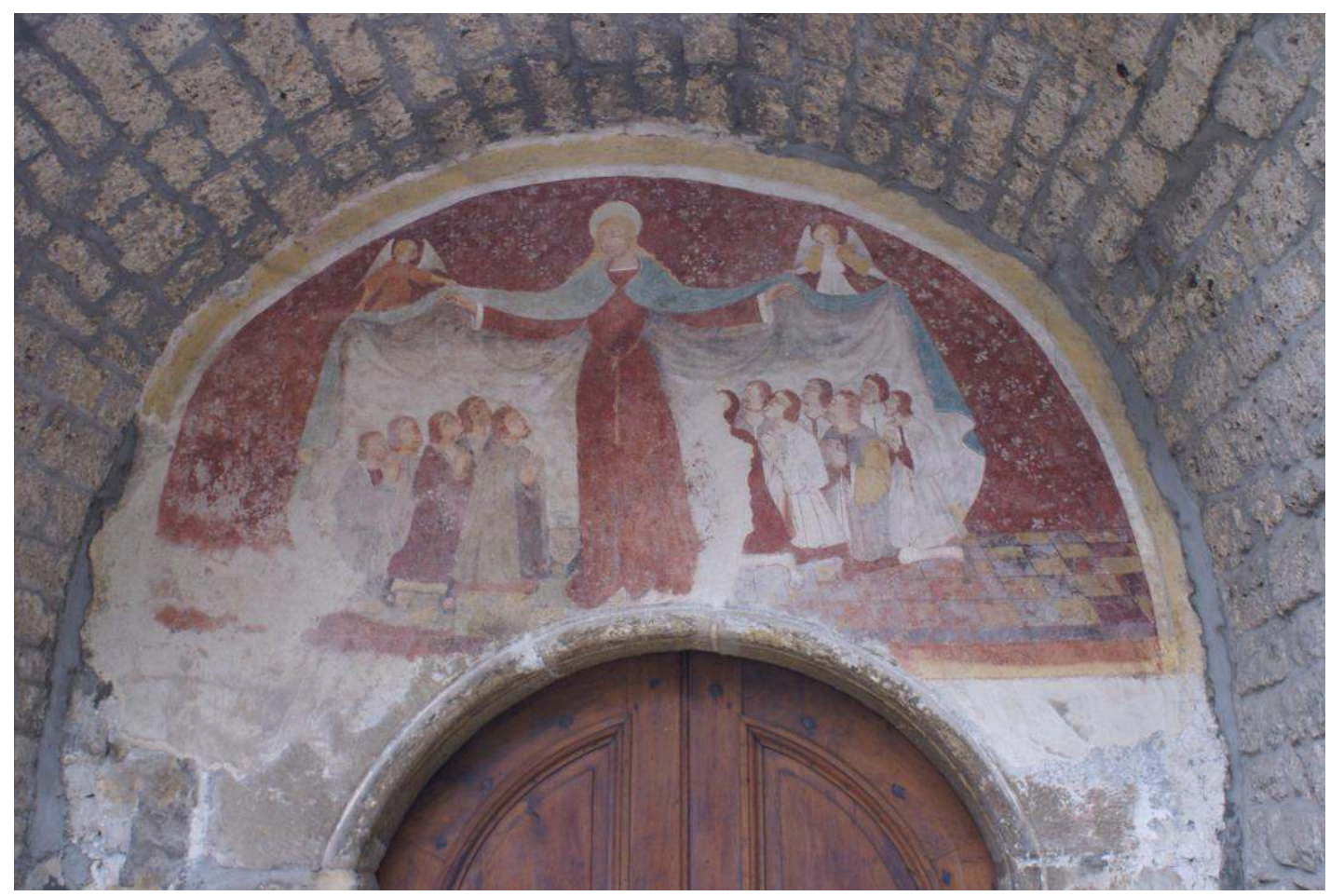

Fig. 3. Vierge au manteau. Peinture murale, a fresco, 170 × 350 cm, ie moitie du xVIe siecle, Anonyme, Église Sainte-Marie, GeneVrey-de-Vif, France. Photographie de l'auteur. 